\title{
PARINAUD'S CONJUNCTIVITIS
}

\author{
BY \\ V. MORAX, \\ OPHTALMOLOGISTE DE L'HÔPITAL LARIBOISIÈRE, \\ PARIS
}

THE aetiology of this peculiar form of conjunctivitis, known by the name of the eminent French clinical master who was the first to describe and to classify the disease, has always excited the attention of workers in our speciality.

I propose to give here a brief statement of the researches that have been made at the Lariboisière Hospital, not that these researches have thrown any light on the nature of the infective agent in Parinaud's conjunctivitis, but rather with the view of putting forward a few facts which prove beyond discussion that Parinaud's conjunctivitis and tuberculous conjunctivitis, two diseases which have led to confusion in the mind of a few ophthalmologists, are distinct morbid entities, and in no way related to one another.

I shall first avail myself of this opportunity to bring to a clear understanding a point in the history of Parinaud's conjunctivitis. In the BRITISH JoURNAL OF OPHTHALMOLOGY, October, 1917, in an article about the death of Professor Goldzieher, I note this sentence : "He was probably the first to describe the affection now commonly. known as Parinaud's conjunctivitis, etc. . . ." It is easy to show that the case of "Lymphoma of the Conjunctiva," published by Goldzieher in 1882, as well as the two other cases published by him in 1893, have no claim to the description of Parinaud's conjunctivitis, and should in no way be considered as identical with this disease. It is quite true that they have all a few clinical features in common; but when we come to the bacteriological and histological examinations, the difference, as regards the infective agent and the reactionary changes, becomes obvious. In a third paper, Goldzieher publishes the histological examination of his three cases, and we note that what he describes corresponds exactly to the follicular type of tuberculous conjunctivitis without ulceration. The presence of giant cells in the middle of infiltrated nodules is by itself a fact which justifies the elimination of these three cases from those described by Parinaud. Had Goldzieher gone further in his investigations, by practising inoculations on the guinea-pig, he would no doubt have convinced himself that he was going astray when he wrote, next to the heading of his third paper, "Conjonctivite de Parinaud" as synonymous with it. But, after all, from what I have said above, the histological examination alone is quite sufficient to differentiate between Parinaud's conjunctivitis and tuberculous conjunctivitis.

Parinaud was well acquainted with tuberculous conjunctivitis, and 
he never failed to have inoculations made whenever he suspected the tuberculous nature of a lesion. In a series of cases published by his former pupils (Sanz, Chaillous, Morax) inoculations on guinea-pigs with a piece of conjunctival tissue, or of pus derived from infected glands, gave negative results, and, together with Chaillous, I have shown that the histological examination of the conjunctiva never revealed the presence of giant cells; whereas in tuberculous conjunctivitis the presence of these structures was constant. And so also was the positive result we obtained by inoculations on guinea-pigs with a piece of the conjunctiva or with pus, either subcutaneously or intra-peritoneally. I am quite aware of the fact that a differential diagnosis between Parinaud's conjunctivitis and tuberculous conjunctivitis, by their clinical features alone, is often a delicate procedure. The error is much the more common as it is customary to say that tuberculous conjunctivitis is always accompanied by ulcerations. Cases, however, where we fail to notice any ulceration are by no means exceptional, and these are precisely the cases that are mistaken for Parinaud's conjunctivitis. Quite recently I had to attend to a girl 15 years of age who had been brought to me by a colleague as an instance of Parinaud's conjunctivitis. I must admit that clinically the mistake was possible, and I reserved my diagnosis until I had made both the histological and the bacteriological (inoculation) examinations. The case proved to be one of tuberculous conjunctivitis.

It is always easy to cut out a small piece of the conjunctiva, half of which is used for inoculation and culture, and the remaining half for histological examination. If the histological examination shows giant cells, we have to deal either with a case of tuberculosis or with one of sporotrichosis. In the first case inoculation will clear up the diagnosis, and in the second, a culture on usual media will show the typical cultural characters of the fungus. If the slide does not show giant cells, the differential diagnosis will have to be made between Parinaud's conjunctivitis and specific disease. In such a case experimental inoculation will prove negative. Microscopical examination may or may not show the spirochaeta pallida, and even in cases of undoubted syphilis, we must say, it is not always possible to find the infective organism.

I have said above that inoculation of small pieces of the conjunctiva or of pus removed from the infected lymphatic glands in the neighbouring parts, did not produce any lesion if these had been derived from a case of true Parinaud's conjunctivitis. In a similar case all attempts to inoculate the guinea-pig had failed. We inserted a small piece of that conjunctiva in the subconjunctival tissues of a chimpanzee. Again we failed to produce the smallest lesion on that animal. The clinical evolution of the disease showed later that in this case we were not dealing with a tuberculous 
affection in any of its clinical varieties, either attenuated, or even of the bovine type which Musy has tried to put forward.

It is a fact that we have not been fortunate enough up to now to discover the infective agent of Parinaud's disease. But this is not astonishing, so numerous are the diseases where the infective agents are still to be discovered, and we do not see why Parinaud's conjunctivitis and tuberculosis of the conjunctiva should be taken as one, just because they have a few clinical features in common. It would be more scientific, we think, to admit that the clinical features by themselves are not always sufficient to clear up differential diagnosis, and we should rather trust the experimental or histological examination. whenever we meet with a conjunctival affection with involvement of the lymphatic glands. The diagnosis of Parinaud's conjunctivitis should be reserved for those cases where the absence of giant cells, and the negative result of inoculation and culture would justify the elimination of tuberculosis, sporotrichosis, etc., of the conjunctiva.

\title{
TRACHOMA IN THE STRAITS SETTLEMENTS
}

\author{
BY \\ J. KIRK, M.D.Edin., \\ OCULIST TO THE EDINBURGH NATIONAL SERVICE MEDICAL BOARD; \\ FORMERLY VISITING OPHTHALMIC SURGEON, GOVERNMENT HOSPITALS, \\ PENANG, STRAITS SETTLEMENTS.
}

TRACHOMA is the most common and widespread of all the eye affections met with in this part of the world. As the result of twenty years' experience, I should say that the three main causes of blindness among the native population are in the order of their occurrence-trachoma, syphilis, and gonorrhoea, and of the three, trachoma is roughly responsible for 70 per cent. In addition, the amount of eye-maiming and disability short of blindness which it causes is enormous, and not realized. I should like in this paper to bring forward a few personal observations which may be of some interest, though I cannot claim any special originality for them.

Trachoma in the Straits is a disease of the towns, and not so much of the country. It is much more common among artizans, clerks, and the shopkeeping class than in agriculturalists or outdoor workers. There also appears on the surface to be a distinct race susceptibility: the great majority of cases met with are in Chinese. Among the Malay population the ratio of occurrence is very much smaller, and the same may be said of the Tamil or Kling, who come from India in their thousands to this prosperous colony. This great frequency of the disease among the Chinese living in a country 